\title{
Preliminary Pre-Clinical Results and Overview on PET/MRI/Fluorescent Molecular Imaging
}

\author{
Keon Wook Kang* \\ Department of Nuclear Medicine, Seoul National University College of Medicine, \& Cancer Research Institute, Seoul \\ National University, Seoul, Republic of Korea
}

\begin{abstract}
Molecular imaging is used for earlier detection, characterization of disease and an earlier assessment of treatment efficacy through imaging molecular/cellular events in living organisms. Fluorescent materials, radioisotopes and MRI enhancers as imaging probes, are being used for optical imaging, PET and MRI respectively. Each imaging modality has advantages and limitations regarding sensitivity, resolution, and tissue penetration of signal. Triple modal imaging combining PET/MRI/fluorescent probes will overcome the limitations of each modality. Recent advances in nanobiotechnology have identified many candidate probes for medical applications. Nanoparticles are able to carry fluorescent dyes, radioisotopes, drugs, genes, and targeting biomarkers. Triple modal nanoparticles could apply to sentinel node imaging by combined PET/MRI scanner for surgical plan and then to sentinel node detection by surgeons' view. Furthermore, if targeting function is added to multimodal nanoparticles as molecular probes, they are able to be used for diagnosis in vitro and in vivo, as well as therapeutic purposes. If nanotechnology and molecular imaging is combined, it could guide surgeons for minimal invasive but accurate surgery and physicans for personalized target therapy by validating the targeting efficiency using multimodal in vivo imaging.
\end{abstract}

Keywords: nanotechnology, molecular imaging, multimodal in vivo imaging.

Molecular imaging is used for earlier detection, characterization of disease and an earlier assessment of treatment efficacy through imaging molecular/cellular events in living organisms. Fluorescent materials, radioisotopes, and MRI (magnetic resonance imaging) enhancers can be used for optical imaging, PET (positron emission tomography) or MRI. Since each modality has its own advantages and limitations, single modal imaging method does not provide optimal solutions for the issues of sensitivity, resolution, tissue penetration of signal and etc (Table 1). PET or MRI has been actively used in clinics for neurologic, cardiologic, and oncologic diagnosis. PET has high sensitivity but poor spatial resolution, and it provides poor anatomical information. MRI has high spatial resolution but poor sensitivity. Advances in clinical imaging are being made through combinations of different imaging modalities, such as PET/CT and PET/MRI. Fluorescent signal has poor tissue penetration, but can be detected by a videoscope during medical or surgical procedures, or by a fluorescent microscope in subcellular level [1]. Multimodal technique combining PET/MRI/optical imaging will overcome the limitations of each modality.

Recent advances in nano-biotechnology have identified many candidates of molecular probes for medical applications. Nanoparticles are able to carry fluorescent dye, radioisotope, drugs, genes, and targeting biomarkers on their surface and inside. These multifunction nanoparticles as

*Address correspondence to this author at the Department of Nuclear Medicine, Seoul National University College of Medicine, 101 Daehangro Jongno Seoul 110-744, Republic of Korea; Tel: +82-2-2072-2803; Fax: +82-2-745-7690; E-mail: kangkw@snu.ac.kr multimodal molecular probes can be used for diagnostics both in vitro and in vivo, as well as therapeutic purposes.

Table 1. Characteristics of Imaging Modalities

\begin{tabular}{|c|c|c|c|}
\hline & Sensitivity & Resolution & Tissue Penetration \\
\hline \hline PET & $\mathrm{pM}$ & $1 \sim 7 \mathrm{~mm}$ & not matter \\
\hline MRI & $\mu \mathrm{M}$ & sub $\mathrm{mm}$ & not matter \\
\hline Fluorescent & $\mathrm{nM}$ to $\mathrm{pM}$ & $\begin{array}{c}\text { in vivo: } \mathrm{mm} \text { to } \mathrm{cm} \\
\text { in vitro: } \mu\end{array}$ & $1 \sim 2 \mathrm{~cm}$ \\
\hline
\end{tabular}

\section{NANOPARTICLES AS MULTIMODAL MOLECULAR PROBES}

Nanotechnology can control the size of materials in nanoscale $(1-100 \mathrm{~nm})$ and their unique chemical and physical properties. Some nanoparticles such as quantum dots exhibit fluorescence with different colors according to their size. A nanoparticle can carry fluorescent dyes inside or on their surface. Nanoparticles containing iron oxide can emit magnetic resonance signal under high magnetic field. If nanoparticles labeled with radioisotopes, they can be detected by gamma cameras or PET scanners. Nanoparticles can handle multiple chemical properties and has a potential for multifunctional and multimodal properties.

Several nanoparticles were developed for MRI/optical dual imaging. One of the examples is superparamagnetic iron oxide (SPIO) nanoparticles linked with near infrared (NIR) fluorescent dye Cy5.5 [2]. SPIO enhances the T2weighted MRI signal. Crosslinked iron oxide nanoparticles (CLIO) have been widely used for MRI. They are coated by 
hydrophilic polymer and biocompatible. NIR optical imaging has low absorption by the tissue, and is less affected by autofluorescence.

Lee et al. developed approximately $45 \mathrm{~nm}$ sized hybrid nanoparticles of a dye-doped silica core and iron oxide satellites $\left(\left(\mathrm{DySiO}_{2}-\left(\mathrm{Fe}_{3} \mathrm{O}_{4}\right) \mathrm{n}, \mathrm{n}=10 \pm 2\right)\right.$ [3]. They conjugated rhodamine-dye-doped silica nanoparticles with water soluble iron oxide by using sulfosuccinimidyl-(4-N-maleimidomethyl)cyclohexane-1-carboxylate (sulfo-SMCC, Pierce) cross-linkers. These dual functional nanoparticles had "coresatellite" structured comprised of a dye doped silica core and multiple satellites of magnetic nanoparticles. A significant improvement in the MRI signal was derived by synergistic magnetism of multiple iron oxide satellites surrounding a core silica nanoparticle and enhanced fluorescence exhibited from rhodamine dye. Finally they conjugated hybrid nanoparticles with HmenB1 antibodies through sulfo-SMCC conjugation and demonstrated simultaneous optical and MR imaging of neuroblastoma cells expressing polysialic acids (PSAs).

Yoon et al. produced $60 \mathrm{~nm}$ sized MRI/fluorescent dual functioning silica-coated core-shell magnetic nanoparticles incorporating organic fluorescent dyes, rhodamine B isothiocyanate (RITC) or fluorescein isothiocyanate (FITC) inside [4]. Silica blocked the exposure of metal ion on the surface of nanoparticles and prevents metal toxicities in cells. Furthermore, incorporation of a fluorescent dye into the silica shell showed the significant increase in photochemical stability, resulting in minimal photobleaching and the increase of fluorescence intensity due to "caging effects" [5]. They added PEG (polyethylene glycol) moiety on the surface of the core-shell nanoparticles to enhance biocompatibility. When they incubated the nanoparticles with the MCF7 breast cancer cell line, they were readily internalized into cytoplasm which can be applied for cell trafficking in vivo. They conjugated anti-Her2 antibody on the surface of nanoparticles using amine moiety for targeting cancer expressing Her2. When they incubated with these nanoparticles with MCF7 cells, they were attached on the surface of cells which can be applied for cancer specific targeting imaging or drug delivery in vivo.

Recently, multifunctional nanoparticle which can be used in four different modalities was developed. Hwang et al. reported a multimodal nanoparticle imaging system that is capable of concurrent fluorescence, bioluminescence, bioluminescence resonance energy transfer (BRET), PET and MRI in vivo in mouse model [6]. They developed a cobalt-ferrite nanoparticle surrounded by rhodamine was conjugated with luciferase and p-SCN-bn-NOTA (2-(4isothiocyanatobenzyl)-1,4,7-triazacyclonane-1,4,7-triacetic acid) followed by ${ }^{68} \mathrm{GaCl}_{3}$. The multifunctional nanoparticles were first incorporated into C6 cells after incubation, and then injected in the thighs of normal nude mice intramuscularly and subcutaneously. Optical bioluminescence, fluorescence, PET and MRI of C6 cells were acquired from the thighs of mice. In this study, quadruple imaging probes successfully showed the simultaneous visualization of four different imaging modalities in the same mouse.

\section{IN VIVO APPLICATIONS OF MULTIMODAL MOLECULAR IMAGING}

If nanoparticles are injected into tissue, they are drained through lymphatic vessels and engulfed by macrophage in the lymph nodes. This characteristic, radiolabeled colloid nanoparticles have been used in clinics to discover the draining lymph node (sentinel node) from the cancer tissue, especially in breast cancer and melanoma [7]. If the sentinel node is free from tumor involvement, the extent of surgical exploration can be reduced and post-operative morbidity will be also reduced. Since sentinel node detection is using the nonspecific characteristic of nanoparticles (not targeting), many different particles were tried in vivo.

Kim et al. reported on in vivo imaging of lymphatics using a near-infrared quantum dots in order to detect the sentinel lymph nodes in a pig model [8]. Fluorescent signal from quantum dots are brighter than organic phosphorus and will not bleach out even under bright surgical light. This lymphatic probe is attractive as it can be visualized by a videoscope during surgery. Despite their potential impact, the clinical application of quantum dots has continuously been claimed for potential toxicity of cadmium.

We used silica nanoparticles which is a non-toxic inert material to examine the feasibility of fluorescent imaging for sentinel lymph node detection [9]. For the fluorescent signal, we incorporated rhodamine $\mathrm{B}$ isothiocyanate into silica nanoparticles. We labeled ${ }^{68} \mathrm{Ga}$, a positron emitter on the surface of silica nanoparticle through SCN-NOTA (2-(4'-isothiocyanatobenzyl)-1,4,7-triazacyclononane-1,4,7-triacetic acid) as a chelating agent (Fig. 1). These nanoparticles have a dual function which can be detected in both PET scanner and optical imaging system. The nanoparticles were injected subcutaneously into foot-pad on the fore leg of mice. At 5 min after nanoparticles injection, fluorescent signals were shown in right axillary lymph node as well as injection site of living mice. We can detect fluorescence signal in frozen sections of lymph node under the fluorescent microscope. We expanded dual imaging function of PET/fluorescence into triple PET/MRI/fluorescent function by using silica nanoparticles with iron oxide core. We produced $50 \mathrm{~nm}$ sized amino PEGylated magnetic silica nanoparticles containing near infrared fluorescent dye, NIR-797 isothiocyanate and iron oxide core (TMSN-N50). We conjugated a chelating agent, 2-(p-isothiocyanatobenzyl)1,4,7-triazacyclononane-1,4,7-triacetic acid (SCN-NOTA) to amine on the surface of TMSN-N50. We labeled a positron emitter, ${ }^{68} \mathrm{Ga}$ on SCN-NOTA-TMSN-N50 by adding ${ }^{68} \mathrm{Ga}$ eluted from a ${ }^{68} \mathrm{Ge} /{ }^{68} \mathrm{Ga}$ generator. ${ }^{68} \mathrm{Ga}-\mathrm{SCN}-\mathrm{NOTA}-$ TMSN-N50 nanoparticles were injected subcutaneously into foot-pad on the leg of mice. Whole-body images were obtained using an animal PET/CT scanner, an animal MRI scanner and an in vivo optical imaging system (Fig. 2). We observed triple PET, MRI and fluorescence signal of the axillary and brachial lymph nodes drained from foot-pad in living mice. After we co-registered PET and MRI imaging using fiducial markers, signals from each modality could be defined at the same location. We also observe fluorescence signals ex vivo after dissecting the lymph node. 


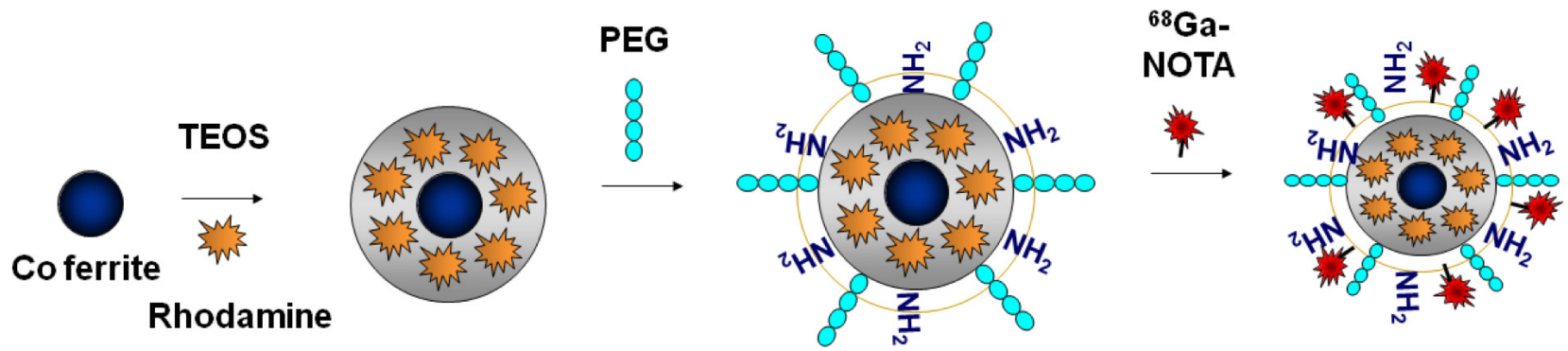

Fig. (1). Schematic diagram of fabricating PET/MRI/fluorescent triple modality molecular probe based on silica nanoparticles with cobalt ferrite core. TEOS; tetraethyl orthosilicate, PEG; polyethylene glycol, NOTA; 1,4,7-triazacyclonane-1,4,7-triacetic acid.
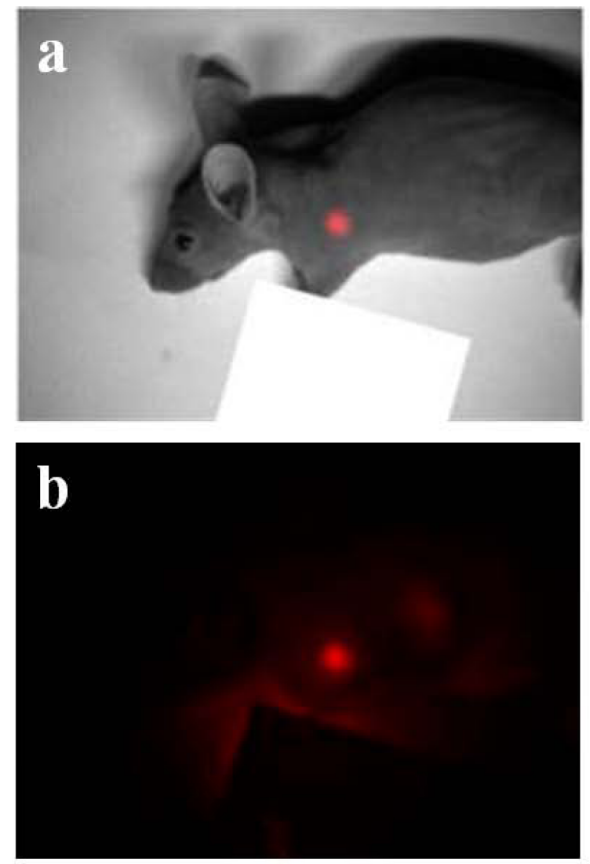
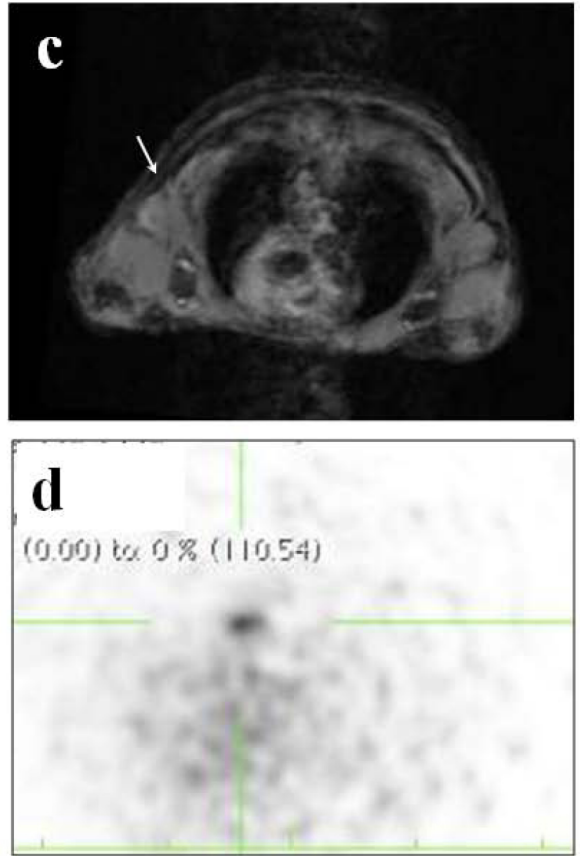
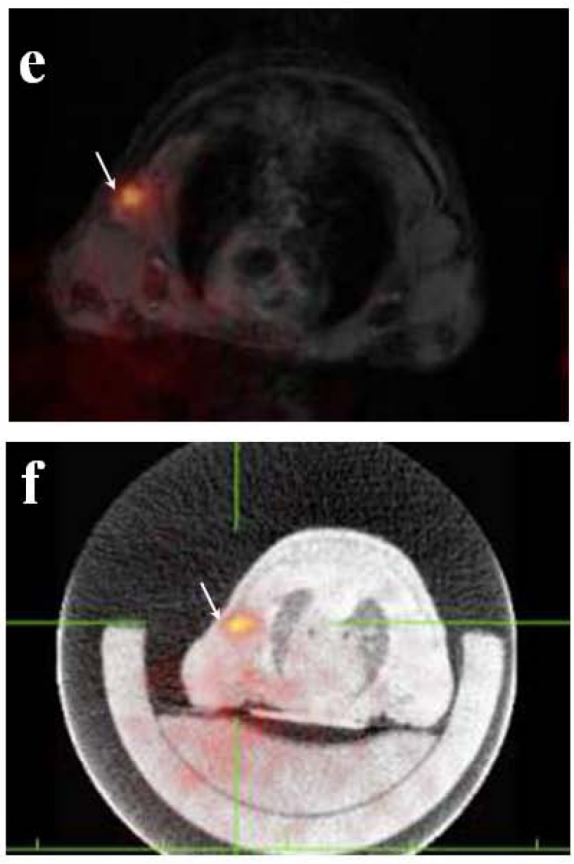

Fig. (2). In vivo PET/MRI/fluorescent triple modal imaging of a mouse injected with ${ }^{68}$ Ga labeled fluorescent nanoparticles. Axillary lymph node was detected by (a, b) Maestro, optical imaging system, (c) MRI and (d) PET (white arrow). PET signal was overlaid on (e) MRI and (f) CT image.

Despite the low amount of radioactivity used for conventional sentinel node detection, concern has been aroused in the nursing and pathology staff [10]. Using a relatively short lived (1 hour) radioisotope ${ }^{68} \mathrm{Ga}$, the lymph nodes can be detected by whole body PET imaging before surgery, and only minimal radioactivity will remain during surgery several hours later. Surgeons can detect the nodes through a videoscope, not using a gamma probe, pathologists can define the nodes using fluorescent microscope.

Stelter et al. modified an aminosilane-coated superparamagnetic nanoparticle for cell labeling and subsequent multimodal imaging using MRI, PET and fluorescent imaging in vivo [11]. They covalently bound the transfection agent HIV-1 tat, the fluorescent dye FITC, and the positron-emitting radionuclide ${ }^{68} \mathrm{Ga}$ to the SPIO nanoparticle. They injected them intravenously into rats, followed by animal PET and MRI. PET showed high uptake in the liver and a faint accumulation in the spleen, but not in other regions. MRI demonstrated a slight hypointensity of the liver. Histological sections revealed green signal in the liver by fluorescence microscopy.
PET is a very sensitive method to visualize the target but reveals poor anatomical information. To overcome this issue, combined PET/CT is widely used in clinics. Recently, combining PET with MRI scanner was actively under investigation, and clinical PET/MRI scanner will be commercially available in the near future [12]. Unlike CT scanner, MRI can detect signals from the nanoparticles as a molecular probe. Although MRI probes are much less sensitive than PET probes, they can be monitored longer not showing the limitation of physical half life of radioisotopes.

PET/MRI/fluorescent triple modality imaging using multifunctional nanoparticles can guide surgical interventions especially minimal invasive procedures such as endoscopic, laparoscopic or robotic ones (Fig. 3). If triple modality molecular probes are used targeting cancer in patients, the locations of tumors and metastatic lymph nodes can be assessed using whole body PET/MRI. The surgeon can locate the tumor, its margin and lymph nodes by fluorescent signal using videoscope during surgery, the pathologist can assess tumor margin and lymph nodes using fluorescent microscope. Finally, postoperative MRI can confirm all the lesions after removal. 


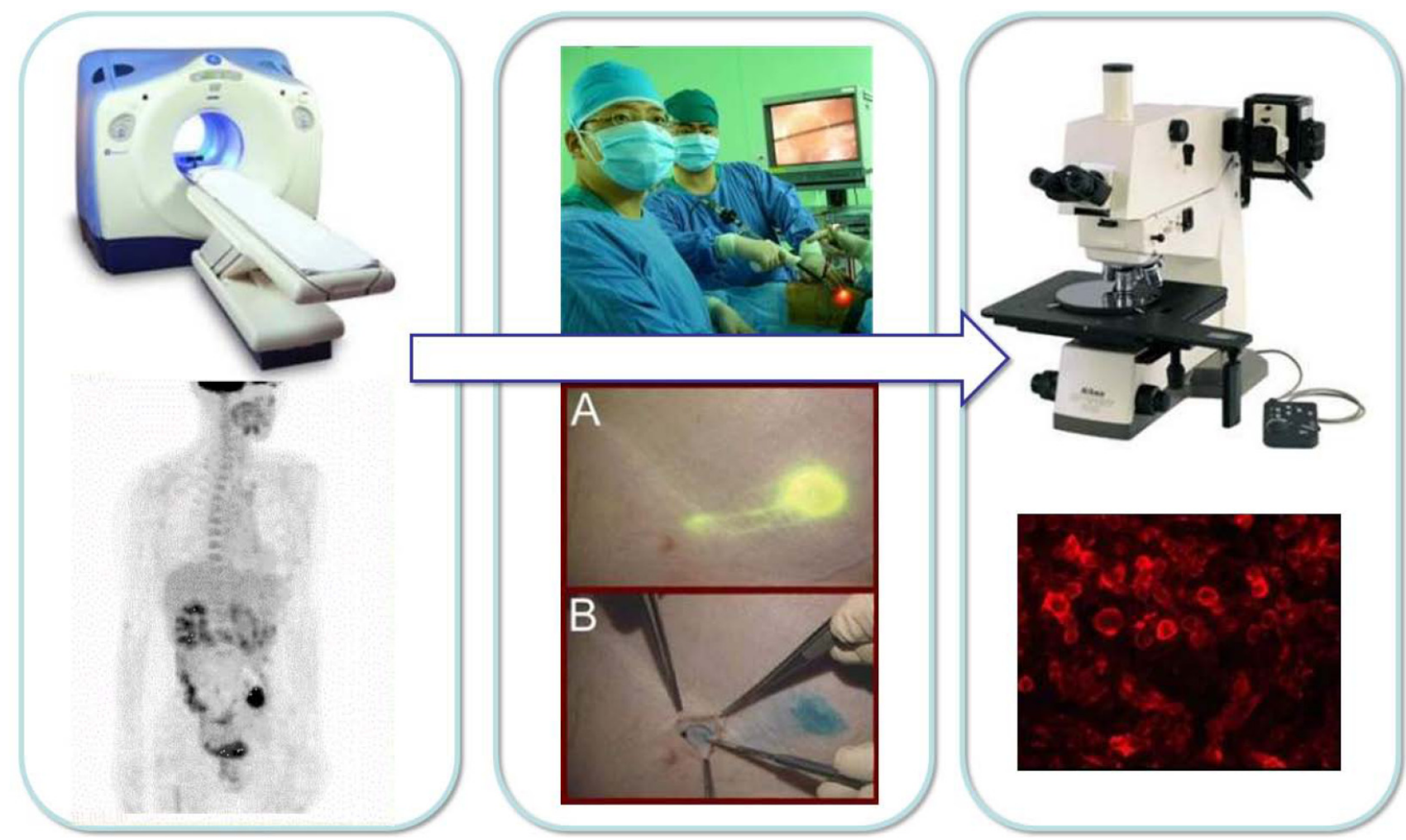

Fig. (3). Surgical guide of cancer treatment using PET/fluorescent dual functioning molecular probe. Functionalized nanoparticles containing fluorescent dye after a promising potential for detecting tumor involvement in the surgical field.

Nanoparticles are able to carry fluorescent dye, radioisotope, drugs, genes, and targeting biomarkers on their surface and inside. We can add targeting function to the triple-modality nanoparticles by conjugating 3D scaffold such as antibodies, peptides, and aptamers. As a result, the particles can trace targets and reveal the lesions after systemic administration. Combining nanotechnology and high throughput proteomics searching for individual biomarkers, personalized targeting therapy might be possible in the future after validating the targeting efficiency using multimodal in vivo imaging.

\section{ACKNOWLEDGEMENTS}

This work was supported by the National R and D Program for Cancer Control (0820320), Ministry for Health, Welfare and Family Affairs, Republic of Korea.

\section{REFERENCES}

[1] Miyashiro I, Miyoshi N, Hiratsuka M, et al. Detection of sentinel node in gastric cancer surgery by indocyanine green fluorescence imaging: comparison with infrared imaging. Ann Surg Oncol 2008; 15: 1640-3.

[2] Kim J, Piao Y, Hyeon T. Multifunctional nanostructured materials for multimodal imaging, and simultaneous imaging and therapy. Chem Soc Rev 2009; 38: 372-90.

[3] Lee JH, Jun YW, Yeon SI, et al. Dual-mode nanoparticle probes for high-performance magnetic resonance and fluorescence imaging of neuroblastoma. Angew Chem Int Ed Engl 2006; 45: 8160-2.
[4] Yoon TJ, Yu KN, Kim E, et al. Specific targeting, cell sorting, and bioimaging with smart magnetic silica core-shell nanomaterials. Small 2006; 2: 209-15.

[5] Ow H, Larson DR, Srivastava M, et al. Bright and stable core-shell fluorescent silica nanoparticles. Nano Lett 2005; 5: 113-7.

[6] Hwang DW, Ko HY, Kim SK, et al. Development of a quadruple imaging modality by using nanoparticles. Chemistry 2009; $15: 9387-93$

[7] Jang SJ, Moon SH, Kim SK, et al. Comparison of the results for sentinel lymph node mapping in the breast cancer patients using ${ }^{99 m} \mathrm{Tc}$ antimony trisulfide colloid, ${ }^{99 \mathrm{~m}} \mathrm{Tc}$-tin colloid, and ${ }^{99 \mathrm{~m}} \mathrm{Tc}$-human serum albumin. Nucl Med Mol Imaging 2007; 41: 546-52.

[8] Kim S, Lim YT, Soltesz EG, et al. Near-infrared fluorescent type II quantum dots for sentinel lymph node mapping. Nat Biotechnol 2004; 22: 93-7.

[9] Jeon YH, Kim YH, Choi $\mathrm{K}$ et al. In vivo imaging of sentinel nodes using fluorescent silica nanoparticles in living mice. Mol Imaging Biol 2010; 12: 155-62

[10] Song YS, Lee JW, Lee HY, et al. Quantitative assessment of the radiation exposure during pathologic process in the sentinel lymph node biopsy using radioactive colloid. Nucl Med Mol Imaging 2007; 41: 309-16.

[11] Stelter L, Pinkernelle JG, Michel R, et al. Modification of aminosilanized superparamagnetic nanoparticles: feasibility of multimodal detection using 3T MRI, small animal PET, and fluorescence imaging. Mol Imaging Biol 2010; 12: 25-34.

[12] Cho ZH, Son YD, Kim HK, et al. A fusion PET-MRI system with a high-resolution research tomograph-PET and ultra-high field 7.0 TMRI for the molecular-genetic imaging of the brain. Proteomics 2008; 8: 1302-23.

This is an open access article licensed under the terms of the Creative Commons Attribution Non-Commercial License (http: //creativecommons.org/licenses/by-nc/ 3.0/) which permits unrestricted, non-commercial use, distribution and reproduction in any medium, provided the work is properly cited. 